\title{
Las encuestas autoadministradas por internet. Un estudio de caso: "las familias adoptivas y sus estilos de vida"
}

\author{
Self-administered online surveys. A case study: "adoptive \\ families and their lifestyles"
}

\author{
María José Rodríguez Jaume y María José GonZÁlez Río \\ Universidad de Alicante \\ mj.rodriguez@ua.es (ESPAÑA)
}

Recibido: 15.12 .2013

Aceptado:28.08.2014

\section{RESUMEN}

España se convierte, en 2004, en el segundo país del mundo en adopción internacional propiciando que la familia adoptiva adquiera una gran visibilidad y relevancia social. El artículo presenta los hallazgos obtenidos a partir de la administración online, a madres y padres adoptivos españoles, de la encuesta Web "Las familias adoptivas y sus estilos de vida". En concreto, se exponen los resultados obtenidos sobre las actitudes, opiniones y percepción de la norma social en materia de adopción. Este análisis permite explorar los factores explicativos que determinan que un subconjunto de la población opte por la adopción al tiempo que nos acerca a la comprensión sociológica tanto del incremento de las adopciones en España como de las familias adoptivas. Adicionalmente, el artículo expone los aspectos técnicos relacionados con el diseño y aplicación de la encuesta on-line con el objetivo de evidenciar que, a pesar de las limitaciones propias de esta forma de aplicación, esta arrojar luz sobre el proceso de adopción internacional, escasamente explorado en la investigación sociológica española con encuestas.

\section{PALABRAS CLAVE}

Adopción internacional, familias adoptivas transnacionales, encuestas online, opiniones frente a la adopción, actitudes frente a la adopción. 


\begin{abstract}
In 2004 Spain becomes the second country in the world for international adoption, favouring a great visibility and social relevance of the adoptive family. The article presents the findings obtained through the administration online to Spanish adoptive parents of the web survey "Adoptive families and their lifestyles". In particular, we expose the results obtained about attitudes, opinions and the perception of the social rule regarding the subject of adoption. This analysis allows us to explore the explanatory factors which determine that a subset of the population chooses adoption at the same time that it approaches us to the sociological understanding both of the increase of the adoptions in Spain and the adoptive families. Furthermore, the article presents the technical aspects related to the design and application of the online survey in order to demonstrate that, despite the typical limitations of this application, this sheds light on the process of international adoption, scarcely explored in the Spanish sociological research using surveys.
\end{abstract}

\title{
KEY WORDS
}

International adoption, adoptive transnational families, online surveys, attitudes for adoption.

\section{INTRODUCCIÓN}

La familia en España, así como en el conjunto de países desarrollados, ha vivido en las tres últimas décadas un profundo proceso de transformación corolario, a su vez, del cambio social y cultural contemporáneo: la postmodernidad. Las alteraciones acaecidas en la institución han alcanzado a todos sus órdenes propiciando la aparición de nuevas conceptualizaciones tales como familia postnuclear, familia postmoderna, familia relacional y familia postpatriarcal. Los cambios que la familia viene experimentando en su estructura, en el proceso de formación (en su doble dimensión de alianza y filiación) y en sus dinámicas de funcionamiento interno, han devenido en un renovado interés académico en la misma de tal modo que hoy es posible consultar una prolija literatura científica que toma como objeto de estudio a las "nuevas formas familiares".

La transición que vive la institución familiar se enmarca, sociológicamente, en el proceso de individualización en el que están instaladas las sociedades más avanzadas (Beck y Beck-Gernsheim 2003) y con el que se explica el mayor protagonismo que asumen, en la cultura contemporánea, las opiniones y comportamientos individuales como consecuencia de la pérdida de control social ante los proyectos familiares (Meil 1999; 2006; 2011). La preeminencia de las opciones individuales conllevaría la transición de la familia nuclear 
(hegemónica y definida a partir de la norma y sanción social de la cultura moderna), hacia las nuevas formas familiares (diversas y definidas a partir de la capacidad electiva o preferencias individuales de la cultura postmoderna).

La sociología de la familia viene vinculando a las nuevas formas familiares (monoparentales, reconstituidas y cohabitantes) con la pluralización de formas, ahora postmatrimoniales, de entrada, permanencia y salida de la vida familiar (Delgado 1993). Esto es, se ha centrado en una de las dimensiones del parentesco: la alianza. Sin embargo, la pluralización de las formas del sistema de filiación, ahora posconsanguíneas, no ha conllevado su inclusión en el catálogo de las nuevas formas familiares (Rodríguez 2012), a pesar de reunir las características (en número y significación) que permiten acuñar de "nuevas" a formas familiares con presencia histórica (Ruiz 2004) ${ }^{1}$.

En España, el fenómeno de las adopciones internacionales irrumpe en la década de los noventa mientras que en Holanda, Francia y países nórdicos su presencia se sitúa en la década de los setenta. A pesar del retraso, su evolución ha sido muy rápida de tal manera que en el año 2004, con 5.541 adopciones en el extranjero, España se convierte en el segundo país del mundo, después de EEUU, en este tipo de adopciones (Selman 2006) ${ }^{2}$. Aunque en los últimos años el flujo de adopciones internacionales se ha reducido, su estudio ofrece la oportunidad de ampliar el conocimiento sociológico sobre las nuevas formas familiares.

El artículo presenta los hallazgos obtenidos a partir de la administración online a madres y padres adoptivos de la encuesta Web "Las familias adoptivas y sus estilos de vida". En concreto, se centra en la exposición de los resultados recabados sobre las actitudes, opiniones y percepción de la norma social frente a la adopción (apartado 4). Este análisis permite explorar los factores explicativos que determinan que un subconjunto de la población adopte a un niño o niña y nos acerca a la comprensión sociológica tanto del incremento de las adopciones en España como de las propias familias adoptivas. Los resultados obtenidos deben contextualizarse en las limitaciones metodológicas y técnicas

${ }^{1}$ Allen P. Fisher (2003: “Still 'Not quite as good as having your own'? Toward a sociology of adoption”, Annual Review of Sociology, 29, pp. 335-361) y más recientemente Peter Conn (2013: Adoption: a brief social and cultural history, New York, Palgrave Macmillan) han subrayado, para el caso norteamericano líder en adopciones internacionales, la escasa presencia de la familia adoptiva como categoría de análisis en las publicaciones académicas vinculadas con la sociología de la familia. Su inclusión en la academia española no difiere de la situación norteamericana descrita. Téngase como ejemplo la reciente incorporación de un capítulo destinado a la familia adoptiva en uno de los últimos manuales dedicados a la familia española (Diana Marre, 2011: "Cambios en la cultura de la adopción y de la filiación”, en Familias. Historia de la sociedad española, Madrid, Cátedra, pp. 893-952).

2 Peter Selman ofrece un análisis demográfico de las adopciones internacionales en quince países para el periodo 1998-2004. En 1998, la tasa bruta de adopciones (número de adopciones por cada 100.000 habitantes) situaba a España en el décimo lugar (su tasa bruta era de 3,8). Seis años después, con una tasa de adopciones de 13 por cada 100.000 habitantes, España pasa a ocupar el segundo lugar, solo superada por Noruega (con una tasa del 15,4). La tasa en 2011 fue de 5,45 adopciones por cada 100.000 habitantes. 
de la investigación. Las lecciones aprendidas en la práctica de encuestas online tienen difícil encaje en una investigación que aborda temas sensibles y para el que se desconoce el marco muestral. La imposibilidad de aplicar un muestreo probabilístico impide la generalización de los resultados pero, como se expone, no invalida el recurso del análisis estadístico con fines exploratorios de la realidad social observada, de ahí que el artículo se detenga en la descripción de las soluciones técnicas adoptadas en el diseño de la encuesta, la selección de la muestra y administración de la encuesta (apartado 3). Los apartados 1 y 2 presentan, respectivamente, el marco teórico y los objetivos e hipótesis que sustentan los resultados que se presentan.

\section{MARCO TEÓRICO.}

\subsection{El estudio sociológico de las adopciones internacionales en España.}

Inicialmente, el interés académico por las adopciones internacionales en España se ha centrado en evaluar aspectos de conducta, adaptación, integración y particularidades médico-sanitarias de las niñas y niños adoptados. Estas líneas de investigación, al desarrollarse desde la psicología y la experiencia clínica, no permiten avanzar en la dimensión sociológica del fenómeno ${ }^{3}$.

Las encuestas sociológicas españolas han incluido de forma marginal el fenómeno de las adopciones. El sondeo de opinión de 1990 sobre "Educación y problemática infantil" introdujo, por primera vez, preguntas relacionadas con el acogimiento y la adopción (Juste et al. 1991). En 2004, el CIS pregunta a la población española su opinión sobre cuestiones vinculadas con los derechos en materia de adopción de las parejas de hecho (CIS 2004a) y de las parejas homoxesuales (CIS 2004a y 2004b). Las encuestas de fecundidad realizadas en España, paradójicamente, incluyen desde 1995 la categoría "hijo/a adoptado/a". En ellas se aprecia el incremento de la paternidad social: en 1995 cinco mujeres declararon tener hijos adoptados mientras que en 2006 fueron cuarenta y cuatro (CIS 1995; Delgado 2007). Por su parte, las estadísticas oficiales que sobre el fenómeno de las adopciones podemos consultar en España no tienen en consideración las recomendaciones que para su registro ofrece Naciones Unidas (2009), lo que imposibilita el conocimiento sociodemográfico del fenómeno y sus protagonistas así como el análisis comparado internacional.

${ }^{3}$ Para una revisión sociodemográfica de las familias adoptivas y adoptantes se pueden consultar los trabajos pioneros de Pere Amorós (1986: La adopción desde una perspectiva socioeducativa, Barcelona, Universitat de Barcelona); Martí March (1993: La adopción en Mallorca: una investigación evaluativa, Palma de Mallorca, Universidad de las Islas Baleares); y Jesús Palacios (1997: La adopción en Andalucía, Sevilla, Consejería de Asuntos Sociales). Para una revisión sociodemográfica de la adopción internacional en España se puede consultar Jesús Palacios et al. (2005: Adopción internacional en España: un nuevo país, una nueva vida, Madrid, Ministerio de Trabajo y Asuntos Sociales). 
La situación descrita para el caso español contrasta con el interés de países como EEUU y Canadá. Estos, desde la década de los noventa, cuantifican el fenómeno de las adopciones a partir de encuestas nacionales realizadas tanto por sus propias administraciones como por institutos y centros de opinión e investigación. La consideración en las dos últimas ediciones del Censo de Población norteamericano (2000 y 2010) de la categoría "hijo/a adoptado/a", ha supuesto un gran avance en el estudio sociológico de la familia adoptiva norteamericana al tiempo que ha impulsado la realización de encuestas nacionales específicas entre las que caben destacar la National Survey of Family Crowth (2002) y la National Survey of Adoptive Parents (2007).

En este contexto, caracterizado por la irrupción del fenómeno de las adopciones internacionales en España y por la escasez de datos cuantitativos (encuestas y/o registros estadísticos), se diseñó la encuesta "Las familias adoptivas y sus estilos de vida" que, a su vez, se inscribe en el estudio "El baby boom de las adopciones internacionales en España. Un estudio sociológico sobre las familias adoptivas y sus estilos de vida"4. Con ella se buscaba aproximarse a la comprensión sociológica de uno de los nuevos tipos familiares que mayor visibilidad y relevancia social ha adquirido en la postmodernidad y para el que el conocimiento sociológico en España es todavía incipiente.

\subsection{Actitudes y opiniones de la población frente a la adopción.}

Hasta la década de los setenta, la adopción ha estado vinculada exclusivamente con la infertilidad (Hoksbergen 1998). Sin embargo, y en el marco del proceso de individualización social, uno de los elementos definitorios con el que se presenta la adopción contemporánea es su desvinculación con la imposibilidad de tener hijos biológicos, circunstancia que ha devenido en la diversificación tanto del perfil de padres, madres y menores adoptados como de las motivaciones subyacentes (Anderson et al. 1993; Malm y Welti 2010). Al convertirse la adopción en una opción electiva de filiación, las familias adoptivas ejemplifican estructuralmente las dos dimensiones paradigmáticas de la familia postmoderna. Por un lado, la pluralización en las formas de acceder a la vida familiar, en donde el matrimonio deja de ser la norma social que rige la alianza. Y, por otro, la pluralización en las formas de tener descendencia, en donde los lazos consanguíneos pierden importancia como norma de filiación.

Una de las líneas de indagación que sobre el fenómeno de la adopción más interés ha suscitado es el estudio de las actitudes y opiniones de la población ante a la adopción. La teoría de la acción razonada (Fishbein y Ajzen 1975) ofrece elementos conceptuales para hipotetizar que las opiniones, actitudes personales y la percepción de la norma social operan como factores explicativos de los comportamientos frente a la adopción de niños y niñas. Los datos disponibles ponen

${ }^{4}$ La investigación fue financiada por el Ministerio de Economía y Competitividad dentro del Plan Nacional de I+D (CSO2009-14763-C03-03). 
de relieve la gran aceptación social que suscita el fenómeno de las adopciones. En Estados Unidos, el 72,5\% de la población compartía actitudes favorables frente a la adopción (Dave Thomas Foundation For Adoption 2013); en Canadá, el 77\% aprobaba la adopción como forma familiar (Miall y March 2005); en Mumbai, el 94\% aprobaba a las familias adoptivas (Raju 1999); y en Japón, el 86,2\% aceptaba la adopción (Mohanty 2013). Para el caso español, Alberdi y Escario han anotado que tres de cada cuatro españoles se imaginan así mismos adoptando (2003: 84).

Sin embargo, y a pesar del reconocimiento social de la familia adoptiva, el número de personas que finalmente concluyen un proceso adoptivo es, relativamente, bajo. Esta brecha, entre actitudes y comportamientos, se viene explicando a partir de las características sociodemográficas de la población con actitudes más positivas hacia la adopción: mujeres, población entre 25 y 64 años, elevada formación, ocupada, de clase social media-alta y posicionada en el espectro político en la izquierda (Dave Thomas Foundation for Adoption 2002, 2013; Van Laningham et al. 2012).

Junto a la identificación de este perfil sociodemográfico, la literatura especializada ha identificado que la percepción de la población sobre las normas sociales asociadas con la ideología pronatalista (Park y Wonch 2013; Van Laningham et al. 2012) y la valoración general hacia los niños (Tyebjee 2003), llevan a que actitudes positivas hacia la adopción concluyan en un comportamiento efectivo. Así, la aceptación y acceso a técnicas de reproducción asistida puede operar como barrera hacia la adopción al tiempo que valoraciones y actitudes más positivas frente a la infancia la propician.

En este contexto, cabe considerar como variables explicativas de los comportamientos efectivos frente a la adopción, los valores postmodernos de la cultura familiar. Meil describe el proceso de transformación que experimenta la vida familiar española en el marco del proceso de postmodernización de la cultura familiar definiéndolo como la mayor tolerancia social hacia comportamientos familiares tradicionalmente estigmatizados (1999: 17). Para Meil, el proceso de pérdida de control social sobre los proyectos de vida familiar se manifiesta con nitidez en la aceptación social y legal de las parejas del mismo sexo y en el reconocimiento de su capacidad para la crianza (2011: 14). En este sentido, la investigación ha desvelado que la población que se identifica con la comunidad lesbiana, gai, bisexual y transgénero (LGBT) muestra actitudes más positivas frente a la adopción (Tybjee 2003). 


\section{OBJETIVOS E HIPÓTESIS.}

El análisis sobre las opiniones, actitudes personales y la percepción de la norma social como factores explicativos de los comportamientos frente a la adopción de niñas y niños, parte de seis hipótesis de indagación que se estructuran en torno a tres objetivos de investigación.

Objetivo 1. Identificar el perfil sociodemográfico de madres y padres adoptivos.

Hipótesis 1.1. La población adoptante comparte una serie de características sociodemográficas que les define y les diferencia respecto a la población no adoptante. Con el fin de contrastar esta hipótesis, la encuesta incluía preguntas de control sociodemográficas.

Hipótesis 1.2. Las madres y padres adoptivos otorgan menos importancia a la descendencia consanguínea (ideología pronatalista). Para la indagación de esta hipótesis, el cuestionario incluyó preguntas dirigidas a: medir la incidencia de los tratamientos de reproducción asistida ${ }^{5}$; las motivaciones que les llevaron a la adopción ${ }^{6}$.

Objetivo 2. Identificar el perfil sociodemográfico de la familia adoptiva.

Hipótesis 2.1. La estructura de las familias adoptivas muestra pluralización en sus formas de acceso a la vida familiar postmoderna.

Hipótesis 2.2. La estructura de las familias adoptivas muestra pluralización en sus formas de filiación, característica de la familia postmoderna.

Para contrastar estas hipótesis el cuestionario recogió preguntas que permiten identificar la estructura familiar a partir de sus rutas de acceso y según estructura de filiación adoptiva o mixta ${ }^{7}$.

Objetivo 3. Identificar valores postmodernos de la cultura familiar.

Hipótesis 3.1. Madres y padres adoptivos otorgan mayor importancia a la vida en familia y a los hijos. Con el fin de contrastar esta hipótesis, el cuestionario incluía preguntas que permitían identificar: la importancia atribuida a la

${ }_{5}^{5}$ Pregunta sección III: ¿Recurrió a técnicas de reproducción asistida o tratamientos de fecundación in vitro?

${ }^{6}$ Pregunta sección VI: Generalmente, la adopción cubre el deseo de una persona o pareja de ser padre o madre pero, ¿qué le motivó a ser padre o madre por la vía de la adopción? (Marque las tres opciones con las que usted se sienta más identificado/a).

7 Definimos estructura de filiación adoptiva a aquellas familias adoptivas que solo cuentan con hijos adoptados. Por su parte, la estructura de filiación mixta identifica a las familias adoptivas que cuentan con hijos biológicos e hijos adoptivos. 
institución familiar ${ }^{8}$; la opinión sobre el papel que cumple la familia en la sociedad actual ${ }^{9}$; y el valor que otorgaban, en general, a los hijos ${ }^{10}$.

Hipótesis 3.2. Madres y padres adoptivos aceptan, en mayor grado que la población en general, las nuevas formas familiares. En concreto, se interrogó sobre la capacidad de crianza de gais, lesbianas y madres solteras ${ }^{11}$.

Con el propósito de alcanzar una mayor compresión sociológica en los objetivos de investigación, los resultados obtenidos en la encuesta fueron comparados con los arrojados por el Barómetro de septiembre de 2010 (CIS 2010) (hipótesis 1.1) y por la encuesta Opinión y actitudes sobre la familia (CIS 2004b) (hipótesis 3.1 y 3.2$)$.

\section{DISEÑO DE LA E-ENCUESTA "LAS FAMILIAS ADOPTIVAS Y SUS ESTILOS DE VIDA".}

El desinterés estadístico del que es objeto el fenómeno de las adopciones en España impide que, a efectos de investigación social, dispongamos de un registro de familias adoptivas desde el que, a modo de marco muestral, se pueda acceder a ellas con el fin de administrar un cuestionario ya sea cara a cara, a través del correo postal o telefónicamente. Sin embargo, en el caso específico de la investigación que se presenta, el universo poblacional objeto de interés -padres y madres adoptantes- es usuario habitual de la red, resultando pertinente y eficaz administrar la encuesta en su modalidad online (Sánchez y Segovia 2008). En torno a la adopción han proliferado sitios Web especializados convirtiéndola en un fenómeno en línea (Wahl et al. 2005: 281). Estos espacios virtuales ofrecen no solo información sino que se presentan como fuentes excepcionales en donde encontrar consejo y apoyo emocional. Ejemplifica la situación señalada el intercambio de 4.103 emails entre el 18 de marzo y el 9 de junio de 2003 en la lista de distribución de AFAC (Marre 2004) ${ }^{12}$. Recientemente, el instituto Evan

\footnotetext{
${ }^{8}$ Pregunta sección I: Utilizando una escala de 0 a 10, en la que 0 significa 'nada importante' y

${ }^{9}$ Pregunta sección IV: Y para usted, personalmente, ¿qué papel cumple la familia? (Seleccionar máximo tres opciones)..

${ }^{10}$ Pregunta sección IV: En general, cuando se refiere a su familia, ¿en qué persona/s piensa concretamente?

${ }^{11}$ Pregunta sección VI: En relación con la adopción de hijos por parejas homosexuales (parejas del mismo sexo) y monoparentales (mujeres y hombres que son madres y padres sin pareja o cónyuge), hay distintas opiniones. ¿Podría indicar su grado de acuerdo sobre las siguientes opiniones? (Pregunta que sitúa las respuestas en una escala de 1 a 4 . Los porcentajes que se indican corresponden a la suma de 1 -muy de acuerdo- y 2 -bastante de acuerdo-).

${ }_{12}$ Marre señala que las relaciones establecidas por las familias adoptantes son, esencialmente, virtuales dado que la mayoría de asociaciones de familias adoptivas no cuentan con sedes físicas. El intercambio de información a través de sus canales de comunicación online (páginas web, listas de distribución y chats) se incrementa en situaciones excepcionales. Así sucedió en 2003 cuando la administración china pospuso la asignación de menores hasta garantizar que el brote de neumonía asiática (SARS) hubiera remitido. En ese contexto, AFAC (Asociación de Familias Adoptantes en China) mantuvo informados a sus miembros a través de los contactos que estableció con asocia-
} 
B. Donalson Adoption ha iniciado una línea de investigación con la que persigue sensibilizar a políticos, profesionales, madres, padres e hijos adoptados sobre el impacto que produce internet (legales, éticos y personales) tanto en el proceso de adopción como en las vidas de las miles de personas a las que alcanza (Howard 2012).

La realización de una encuesta implica el desarrollo de una serie de etapas que han de seguirse con independencia de la forma en la que se administre el cuestionario. No obstante, las encuestas en Internet presentan particularidades que justifican una atención diferencial, especialmente, en las siguientes fases (Couper 2000): muestreo y selección de los encuestados, diseño de la encuesta, distribución y gestión de la respuesta, realización de la encuesta piloto y registro de datos.

\subsection{Diseño muestral.}

La calidad de la muestra es uno de los elementos en los que se fundamenta la eficacia de la encuesta. La pretensión de acceder a muestras muy grandes no garantiza el éxito de la encuesta y no debe tomarse como sustituto de muestras precisas (Baatard 2012). Un medio que hace posible llegar a más gente no debe ignorar los procedimientos de muestreo más apropiados (Couper 2000).

En el caso que nos ocupa, el universo poblacional queda constituido por el subconjunto de madres y padres españoles que han pasado por la experiencia de la adopción internacional. A diferencia del muestreo específico que se puede aplicar en grupos organizados (Rodríguez 2005), se desconocía el marco muestral desde el que contactar directamente con el universo: no existe un registro centralizado de padres adoptivos y los organismos que en cada Comunidad Autónoma gestionan los procesos de adopción no cedieron los datos identificativos de las posibles unidades muestrales dado que están sujetos a la Ley de Protección de Datos. Desde la consideración de que la adopción se ha convertido en un fenómeno en línea, la encuesta fue diseñada para ser autoadministrada en la Web desarrollando, siguiendo a Couper (2000), un mиestreo no probabilístico por autoselección.

La invitación a participar en la encuesta suscitó el interés de 834 madres y padres adoptivos (número de personas que accedieron a la página de presentación del cuestionario). De estas, 385 iniciaron la cumplimentación del cuestionario y 239 lo finalizaron. Nueve cuestionarios no superaron las preguntas de consistencia incluidas en el cuestionario, obteniendo una muestra final de 230 madres y padres adoptivos.

ciones similares en Estados Unidos y Canadá y con la información que diariamente procedía de China por mediación de un traductor. 


\subsection{Invitación, recordatorio e incentivos.}

En una encuesta electrónica la tasa de respuesta aparece condicionada por: el modo, contenido y formato de la invitación; el número de contactos o recordatorios realizados; y los incentivos ofrecidos (Sánchez y Segovia 2008). Respecto al primero de los aspectos, se aplicó un protocolo de invitación múltiple (Couper et al. 2001; Yun y Trumbo 2000) con el que se perseguía llegar al mayor número de familias adoptivas y garantizar tanto la privacidad y anonimato de los participantes como la confidencialidad de los datos recabados. Estos últimos aspectos, frecuentemente obviados en la investigación con encuestas online, son relevantes también por sus implicaciones éticas.

La primera invitación a colaborar con la investigación se cursó a las entidades que en las Comunidades Autónomas gestionan el proceso de adopción. Estas declinaron colaborar argumentando que carecían de recursos humanos para asumir el rol de informantes clave. Ante esta circunstancia, debíamos buscar una vía de acceso a padres y madres adoptivos. En nuestro ámbito de estudio se podrían haber utilizado las listas de correo de libre acceso en la red. Sin embargo, Cho y LaRose (1999) advierten contra su uso, especialmente de aquellas que han sido creadas por internautas con el propósito de crear redes de información y apoyo emocional. Su utilización por parte de personas ajenas a la red es percibida por los integrantes como una intromisión a su intimidad. Contactamos entonces, con las asociaciones de familias adoptivas españolas (treinta y nueve) invitándolas a participar en la investigación. La práctica totalidad de las asociaciones que participaron en la investigación manifestaron su compromiso a lo largo de una semana y todas lo hicieron a través del correo electrónico. Transcurridos diez días se mandó una segunda invitación, a modo de recordatorio, a las asociaciones que no contactaron con el grupo de investigación después de la primera invitación. En esta segunda etapa dos asociaciones más se adhirieron al proyecto. Finalmente, diecinueve asociaciones de familias adoptivas colaborando en la investigación, circunstancia que favoreció que se diera a conocer la encuesta en todas las comunidades autónomas.

En una tercera etapa se envió a las asociaciones de familias adoptivas colaboradoras la invitación que debían dirigir a sus asociados a través del correo electrónico. En esta invitación se incluía la URL que daba acceso a la encuesta. Para paliar los problemas de privacidad e incrementar la confianza de los padres adoptantes en la investigación, tanto en la página web del grupo de investigación como en la primera página del cuestionario se indicaba la identidad del grupo, se daba la referencia del contacto, se ofrecía información sobre los objetivos de la investigación y se exponían los criterios que iban a garantizar el anonimato y confidencialidad de los datos recabados (ver Figura 1). La dirección IP del ordenador con la que se accedía al cuestionario sólo se utilizó para contrastar que no se hubieran cumplimentado cuestionarios con la misma dirección. 


\section{FIGURA 1. Página Web grupo de investigación}

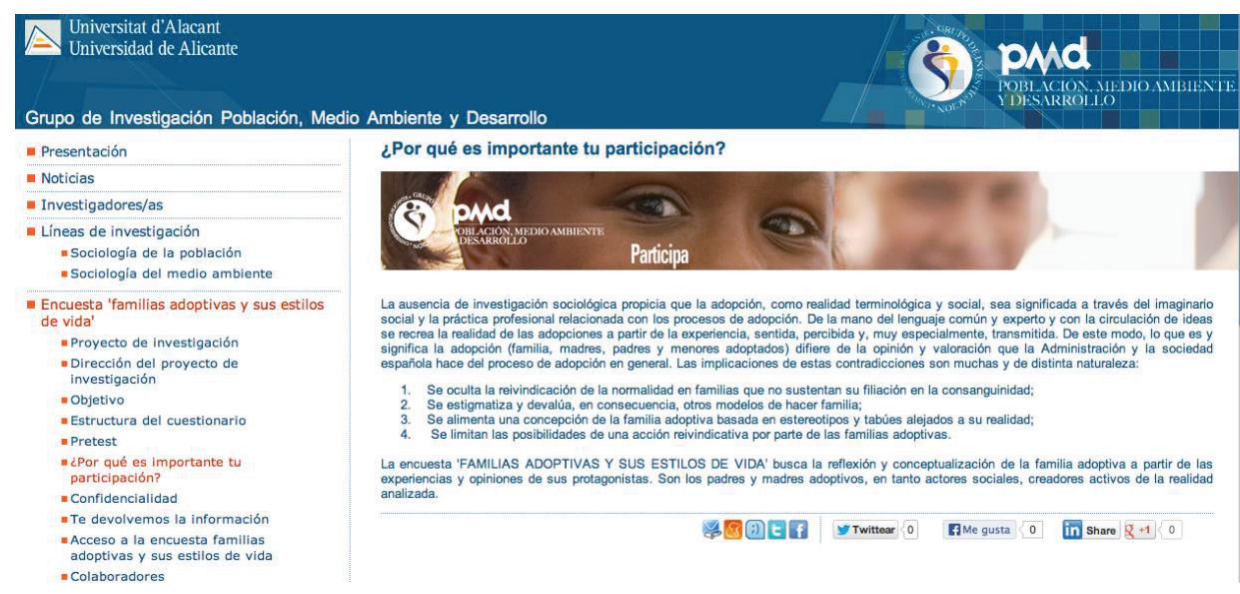

Fuente: encuesta Web Las familias adoptivas y sus estilos de vida

Respecto al recordatorio, este es eficaz cualquiera que sea la modalidad de aplicación del cuestionario. En la encuesta que nos ocupa, el contacto se realizó exclusivamente a través de los responsables de las asociaciones de familias adoptivas. En los meses que la encuesta estuvo activada (julio, agosto y septiembre de 2012) el equipo de investigación se puso en contacto con ellos en dos ocasiones para que alentaran la participación entre sus asociados.

Por último, la investigación ha demostrado que el uso de pre y post-incentivos incrementan la tasa de respuesta en encuestas Web (Cho y LaRose 1999; Couper 2000; Dillman et al. 2009; Groves et al. 1992; Sánchez y Segovia 2008), si bien Bosnjak y Tuten (2003) han señalado que cuando estos son monetarios son más eficientes a través de un sorteo que asignándolos individualmente. En el caso que nos ocupa, se alentó la participación a través del sorteo de un iPad entre quienes cumplimentaran la totalidad del cuestionario. Este post-incentivo se anunciaba en la primera página del cuestionario indicando que el sorteo se realizaría una vez finalizado el proceso de recogida de información.

\subsection{Diseño de la e-encuesta.}

En los cuestionarios administrados a través de la Web, el diseño del cuestionario reviste especial interés pues debe de sumar a las consideraciones de carácter general, comunes a cualquier tipo de aplicación, las singularidades que introduce Internet (Couper 2000; Couper y Miller 2008; Dillman et al. 2000; Ganassali 2008; Gräf 2000; Lozar et al. 2002; Shropshire et al. 2009).

El cuestionario se estructuró en torno a ocho secciones temáticas: variables sociodemográficas (I); familia de origen (II); relación de pareja (III); familia 
de procreación (IV); ideas y valores en torno a la familia $(\mathrm{V})$; proceso familia adoptiva (VI); familia interracial (VII); y satisfacción vida familiar (VIII). Las secciones I, II, III, V y VIII operacionalizaban los conceptos y las dimensiones analíticas que desde la sociología se viene aplicando en el estudio de la familia. Las preguntas incluidas tomaron como referencia las encuestas que el Centro de Investigaciones Sociológicas realiza en ámbitos como la infancia, familia, maternidad, roles de género e inmigración. Del conjunto de preguntas incluidas en el cuestionario, el 29,2\% procedían del banco de indicadores del CIS. Las secciones IV, VI y VII recogen cuestiones específicas sobre el proceso de adopción. La mayoría de los ítems incluidos en estas secciones son de nueva creación y se enunciaron cumpliendo los criterios de exhaustividad, exclusividad y claridad. Las categorías de respuestas se formularon atendiendo al estudio previo realizado sobre veinte y ocho documentos personales autobiográficos publicados entre 1990 y 2011 por los propios padres adoptivos. Las preguntas se ordenaron siguiendo la lógica inherente a la experiencia adoptiva que viene marcada por las etapas del proceso administrativo: solicitud, idoneidad, espera, asignación y encuentro.

La literatura sobre metodología de la encuesta, recomienda en general, que los cuestionarios sean breves (Baatard 2012; Ganassali 2008; Díaz de Rada 2012). El argumento que sustenta esta recomendación es el de incrementar la tasa de respuesta, esto es, alcanzar tamaños muestrales elevados que compensen los sesgos de observación o cobertura. En el caso particular de las encuestas Web se ha señalado que los cuestionarios largos pueden incrementar la tasa de no respuesta de ítems, y que la calidad de las respuestas a las preguntas situadas al final del cuestionario puede ser menor que las ubicadas al inicio (Couper 2008; Vicente y R Reis 2010). La extensión de una encuesta, por regla general, no debe ser juzgada tanto por el número de preguntas formuladas como por la duración media de la entrevista. Crawford, Couper y Lamias (2001) recomiendan una duración máxima de diez minutos mientras que Gunn (2002) la sitúa en veinte. La duración media de la encuesta que nos ocupa fue de cincuenta minutos. A pesar de su extensión, quienes participaron en el pretest consideraron la experiencia positiva y muy útil porque les daba la oportunidad de dejar constancia de sus propias vivencias y con ello contribuían a que se hablara de las familias adoptivas con más información y veracidad. Los encuestados percibieron el valor y la importancia de la encuesta, aspectos que convierten a las entrevistas largas en experiencias muy gratificantes y satisfactorias (Sheatsley 1983) ${ }^{13}$.

La fase de diseño del cuestionario concluyó con el pretest. Este se realizó siguiendo el proceso de pruebas múltiples desarrollado por Dillman (2000). En una primera etapa el cuestionario fue depurado y validado por expertas del CSIC y especialistas en adopción. Sus aportaciones permitieron adecuar el cuestionario a los objetivos de la investigación así como garantizar la pertinencia de las

${ }^{13}$ P. B. Sheatsley (1983): "Questionnaire construction”, en Handbook of interview research, Orlando, Academic Press Inc., pp. 195-230 (en Ma Ángeles Cea D’Ancona (2004): Métodos de encuesta, Madrid, Síntesis, pp. 286) 
preguntas incluidas. En una segunda etapa se realizó el pretest, reproduciendo las condiciones en las que sería administrado, a seis madres y cuatro padres adoptivos lo que permitió homogeneizar aspectos temáticos y conceptuales, comprobar la secuencia lógica de las preguntas así como omitir el efecto contexto o de orden (especialmente importante cuando se trabaja con distintas páginas) que originaban algunas preguntas. Para comprobar que los errores observados se habían subsanado, una segunda versión del cuestionario fue administrada a seis madres y tres padres adoptivos. Adicionalmente, y en las dos etapas en las que el cuestionario se testó entre la población objeto de estudio, les pedimos que nos dieran una valoración cualitativa sobre el mismo. Como se ha señalado, sus respuestas evidenciaron su satisfacción por participar en una investigación que contribuía a alcanzar un mayor conocimiento sobre la adopción y su modelo familiar. Finalmente, el cuestionario fue revisado por tres personas ajenas a la investigación con la finalidad de identificar errores tipográficos introducidos involuntariamente en las fases anteriores.

\subsection{Aspectos gráficos y visuales de la e-encuesta.}

El cuestionario Web, como cualquier cuestionario auto-administrado, va a conjugar el lenguaje escrito con el lenguaje gráfico (Couper 2001; De Leeuw 2008; Dillman et al. 2000; Ganassali 2008; Tourangeau et al., 2004). No obstante, y dada la naturaleza visual de Internet, las implicaciones del lenguaje gráfico en este tipo de encuestas adquieren mayor protagonismo. Para garantizar que los encuestados accedieran y siguieran el cuestionario en las mismas condiciones y en una situación social similar (Llauradó 2006; Sánchez y Segovia 2008), la encuesta Web fue compatible con distintos navegadores (Explorer, Mozilla, Chrome, Opera, Safari y Netscape), sistemas operativos (Windows, Linux, Mac, FreeBSD y Unix). La resolución mínima de pantalla fue de 800x600 pixel permitiendo su ejecución tanto en dispositivos de escritorio como en móviles.

La encuesta fue diseñada en varias páginas. En la primera se recogía de forma breve el objetivo y finalidad de la encuesta, se incluían instrucciones para su cumplimentación y se daba acceso al resto de páginas en las se organizaban las preguntas adscritas a cada una de las secciones temáticas. El diseño permitía avanzar de una forma muy intuitiva: el encuestado debía cumplimentar las preguntas recogidas en la página y cliquear sobre la opción "continuar". Para progresar en el cuestionario debían haber cumplimentado todas las preguntas incluidas en la página.

En cada una de las páginas Web del cuestionario se introdujo un indicador o barra de progreso general con la que se informaba del punto del cuestionario en el que se encontraban. Se pretendía con ello reducir el efecto cansancio (Ganassali 2008: 23) que introducen cuestionarios extensos, alentar la auto-cumplimentación del cuestionario (Vicente y Reis 2010; Yan et al. 2011) y disminuir la tasa de abandono. Adicionalmente, el encuestado siente que es honestamente informado sobre su duración (Llauradó 2006). 
El diseño del cuestionario permitía la interrupción y reanudación de su autocumplimentación en cualquier momento reproduciendo, de este modo, la misma situación que vive un encuestado cuando autocumplimenta un cuestionario en formato tradicional (Kaczmirek 2005). En el momento en que el encuestado accedía a la primera página del cuestionario, se le indicaba su código de identificación con el que podría acceder a la página en la que hubiera interrumpido la tarea.

Siguiendo las recomendaciones de Couper, Traugott y Lamias (2001), el diseño de la encuesta fue personalizado. En la cabecera de cada una de las páginas se introdujeron imágenes (fotografías) neutras y alegóricas de la adopción asumiendo funciones, exclusivamente, ilustrativas (ver Figura 2).

FIGURA 2. Cabecera de la encuesta 'Las familias adoptivas y sus estilos de vida'. Sección 1, página Web 2

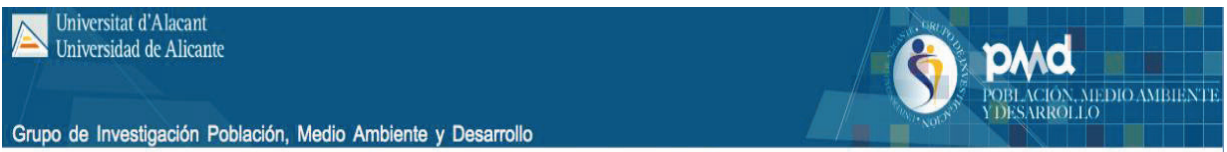

- Presentación

- Noticias

- Investigadores/as

- Lineas de investigación

- Sociología de la población

- Sociología del medio ambiente

- Encuesta 'familias adoptivas y sus estilos de vida'

- Proyecto de investigación

- Dirección del proyecto de

investigación

- Objetivo

- Estructura del cuestionario

- Pretest

- ¿Por qué es importante tu

participación?

- Confidencialidad

- Te devolvemos la información

- Acceso a la encuesta familias

adoptivas y sus estilos de vida

- Colaboradores
¿Por qué es importante tu participación?

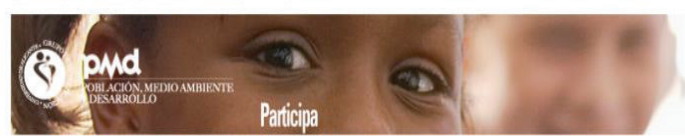

La ausencia de investigación sociológica propicia que la adopción, como realidad terminológica y social, sea significada a través del imaginario social y la práctica profesional relacionada con los procesos de adopción. De la mano del lenguaje común y experto y con la circulación de ideas se recrea la realidad de las adopciones a partir de la experiencia, sentida, percibida y, muy especialmente, transmitida. De este modo, lo que es y significa la adopción (familia, madres, padres y menores adoptados) difiere de la opinión y valoración que la Administración y la sociedad española hace del proceso de adopción en general. Las implicaciones de estas contradicciones son muchas y de distinta naturaleza:

1. Se oculta la reivindicación de la normalidad en familias que no sustentan su filiación en la consanguinidad: Se estigmatiza y devalúa, en consecuencia, otros modelos de hacer familia;

Se alimenta una conceepción de la tamilia adoptiva basada en estereotipos y tabues alejajơos a su realidad,

4. Se limitan las posibilidades de una acción reivindicativa por parte de las familias adoptivas.

La encuesta 'FAMILIAS ADOPTIVAS Y SUS ESTILOS DE VIDA' busca la reflexión y conceptualización de la familia adoptiva a partir de las experiencias y opiniones de sus protagonistas. Son los padres y madres adoptivos, en tanto actores sociales, creadores activos de la realidad

Fuente: encuesta Web Las familias adoptivas y sus estilos de vida

\section{FACTORES EXPLICATIVOS DE LA ADOPCIÓN EN ESPAÑA.}

\subsection{Perfil sociodemográfico de las madres y los padres adoptivos españoles.}

La investigación en adopción realizada con muestras no probabilísticas ha puesto de manifiesto el sesgo de género en las muestras obtenidas. En el caso que nos ocupa se reproduce el patrón de mayor participación de mujeres: un 83,5\% 
de los cuestionarios fueron autocumplimentados por madres adoptivas. La edad media de la muestra fue de 44,5 años: 44 años las madres y 46 años los padres.

Los datos sociodemográficos de la muestra (ocupación, nivel de estudio y estado civil) definen el carácter selectivo de la población adoptiva. El 81\% de la muestra se encontraba trabajando: un 34,3\% como técnicos y profesionales científicos e intelectuales; un 19,1\% desempeñaban empleos de tipo administrativo; y un $11,7 \%$ eran técnicos y profesionales de apoyo. Estos datos contrastan con los arrojados por la población general: $12,2 \%, 4,1 \%$ y 13,9\%, respectivamente (CIS 2010). En los casos en los que el encuestado se encontraba en paro (el $9,1 \%$ ), sus cónyuges o parejas se encontraban trabajando. Esta estructura laboral explica que el $63,5 \%$ de las madres y padres adoptivos, frente al 32,8\% de la población general (CIS 2010), perciba que su situación económica personal era muy buena o buena. La presencia de madres y padres adoptivos con niveles de formación superior triplica a la de la población en general (el 72,7\% frente al 20,7\% de la población en general -CIS 2010), avalando los datos que sitúan a la población con mayores niveles de estudios entre la más favorable a la adopción.

La muestra, mayoritariamente, comparte el estado civil de casado (83,5\%). Estos datos corroborarían los hallazgos previos y que han anotado que las personas casadas no solo muestran una actitud más positivas hacia la adopción (Dave Thomas Foundation for Adoption 2002) sino que es el colectivo que más participa en la adopción internacional (Ishizawa y Kubo 2014). La muestra de adoptantes se identificaría, y diferenciaría respecto al conjunto de la población española, por situarse políticamente en posiciones de izquierdas $(\mathrm{el} 63,6 \%)^{14}$ y por la fuerte presencia del ateísmo (el 42,2\%) (frente al 30,2\% y el 8\%, respectivamente, de la población en general -CIS 2010).

El 49,6\% de la muestra llegó a la adopción porque esta era "su elección". Sin embargo, y dado que la "imposibilidad de tener un hijo biológico" fue la segunda motivación esgrimida (alcanzando al 40,4\% de la muestra) resulta de interés determinar si esta 'elección' deviene de la pérdida de importancia de la consanguinidad como norma de filiación o es una 'elección' ante la imposibilidad de tener hijos biológicos. En este sentido, el 41,9\% de las madres y padres adoptivos que declararon contar con diagnóstico de infertilidad no se sometió a tratamientos de infertilidad con anterioridad a la adopción. Particularmente, entre las familias que solo cuentan con hijos adoptados, este porcentaje asciende y se sitúa en el $55,1 \%$. Esto es, la adopción actualmente se desvincula de la infertilidad para convertirse en una opción de filiación electiva.

14 En ambas encuestas la autoubicación política se presentaba en una escala del 1 al 10. Los resultados indicados corresponden a los valores acumulados en la ubicación de izquierdas (del 1 al 4). 


\subsection{Perfil sociodemográfico de las familias adoptivas españolas.}

En el contexto del estudio de las nuevas formas familiares adquiere especial significación la identificación de la estructura familiar en sus dos dimensiones constitutivas: la alianza y la filiación. Respecto a la primera dimensión, las familias adoptivas no reproducirían la pluralización de formas de acceso a la vida familiar pues, como se ha anotado, el 83,5\% se adscriben al modelo matrimonial. Las familias monoparentales, las parejas que conviven y las familias homoparentales representan, respectivamente, el 10\%, el 1,3\% y el 3\% de la muestra.

No obstante, y dado que la legislación que regula el proceso de adopción en los países de origen de los menores, mayoritariamente excluye a las uniones de hecho, a las familias monoparentales y a gais y lesbianas, se les preguntó si el hecho de haber contraído matrimonio obedecía a la decisión de adecuar sus características familiares a los requisitos legales exigidos. El 14,3\% de los encuestados declaró que contrajo matrimonio para facilitar el proceso de adopción. Esto es, si el estado civil no condicionara el éxito de la adopción, la cohabitación habría representado en la muestra de adoptantes al 15,6\%. Al institucionalizar la relación, las parejas alcanzan un doble objetivo: en primer lugar, les sirve como estrategia de ajuste a las reglas que rigen la adopción internacional; y, en segundo lugar, les permite sortear la marginalidad a la que han sido relegados tipos familiares con estructuras que desafían el modelo normativo (familias monoparentales y homoparentales) y a las que frecuentemente, se les asignan niños con necesidades especiales o difíciles de asignar (Raleigh 2012).

Respecto a la estructura familiar según tipo de filiación, el 76,5\% de la muestra estaba constituida por familias con filiación adoptiva, esto es, solo contaban con hijos adoptivos: el 61,9\% contaban con un hijo adoptado mientras que el $34,1 \%$ habían adoptado a dos hijos. Entre las familias adoptivas de filiación mixta, con hermanos biológicos y adoptados, los segundos llegan, en el 79,6\% de los casos, después de haber tenido hijos biológicos. En el 3,7\% de las familias de filiación mixta los hijos adoptados llegan después de la reconstitución familiar en la que alguno de los progenitores ya contaba con algún hijo biológico.

\subsection{Valores postmodernos de la cultura familiar en las familias adoptivas.}

La familia, a pesar de los profundos cambios que viene protagonizando, continúa siendo una de las instituciones más importantes en la vida de la población. Así lo valoran el $86 \%$ tanto de la muestra de madres y padres adoptivos como la población en general, si bien para los padres adoptivos, la familia es la faceta de la vida a la que mayor importancia le atribuyen, mientras que para la población en general la salud ocuparía el primer lugar (90,1\% de la población) (CIS 2010).

Dos elementos adicionales separan a la muestra de adoptantes de la población en general. Ambos aspectos nos remiten a la constatación de dos características propias de la familia postmoderna: la centralidad que asumen los hijos en la vida familiar (Beck y Beck-Gernsheim 2001) y la sentimentalización de la relación 
paterno-filial (Meil 2006). Respecto al primero de los aspectos, el 90,4\% de los encuestados se refiere a sus hijos cuando piensa en su familia mientras que, en la población en general, este porcentaje se sitúa en un 65,9\% (CIS 2010). Similares diferencias se aprecian cuando ofrecen su opinión respecto a las funciones que, personalmente, creen que desempeña la familia actualmente: el 62,4\% de la muestra manifiesta que es la de proporcionar 'amor y afecto'; porcentaje que se reduce al 38\% entre la población en general (CIS 2004b).

Uno de los aspectos que la investigación en adopciones ha destacado respecto al colectivo más favorable hacia la adopción, es su mayor identificación con la comunidad de lesbianas, gais, transexuales y bisexuales (LGTB) (Tybjee 2003). En este sentido, se aprecian distancias entre la muestra de adoptantes y la población en general. Los primeros apoyan, en un $84,8 \%$, que gais y lesbianas adopten y consideran, un $86,5 \%$, que garantizan el bienestar de los niños. Por su parte, la población en general sancionaría más estos comportamientos: un 42,4\% contemplaría la adopción entre parejas del mismo sexo y un $63,7 \%$ opina que garantizan el bienestar los hijos (CIS 2004b).

\section{CONCLUSIONES}

Los resultados arrojados por la encuesta "Las familias adoptivas y sus estilos de vida" ofrecen una primera aproximación al estudio de la familia adoptiva transnacional, uno de los nuevos tipos familiares que, por su número y significación, mayor visibilidad y relevancia social han adquirido en España en las dos últimas décadas.

Los datos recogidos dibujan el perfil sociodemográfico y familiar de quienes eligen la adopción como forma de filiación y que, en general, no difiere del identificado en la investigación internacional: elevada formación, situación económica buena y estable, sin identificación religiosa y situados a la izquierda del espectro político. Mayoritariamente, las familias adoptivas se adscribirían al modelo matrimonial. Si embargo, se ha constatado que para un grupo de padres y madres adoptivos que cohabitaban, el matrimonio es una estrategia que, en el marco de las normas que regulan la adopción internacional, les permite culminar con éxito su deseo de ser padres a través de la adopción sin sufrir la marginación que experimentan las familias homoparentales y monoparentales.

Una de las características de la estructura familiar que ha desvelado la encuesta es la convivencia, en una de cada cuatro familias encuestadas, de los hijos adoptados con hermanos biológicos. Adicionalmente, entre las familias con solo hijos adoptivos, y en las que sus progenitores contaban con un diagnóstico de infertilidad, menos de la mitad agotó las opciones médicas para tener un hijo biológico. Estas evidencias muestra la desvinculación de la adopción contemporánea respecto a la imposibilidad de tener hijos biológicos, motivación que ha regulado la adopción hasta la década de los setenta. A su vez, y como corolario del proceso de individualización social, los datos muestran la menor importancia atribuida entre los padres adoptivos a la norma de filiación 
basada en la consanguinidad al tiempo que sitúa la adopción como una forma de filiación electiva. A partir de la hegemonía, en la cultura Occidental, de la ideología de la familia genética, estos hallazgos señalan un nuevo elemento de cambio en la familia escasamente abordado ya que el estudio de las nuevas formas familiares se viene desarrollando a partir de los modelos familiares que resultan de la desinstitucionalización del matrimonio.

La identificación de las características sociodemográficas compartidas por madres y padres adoptivos permite no solo identificar su perfil sino que propicia el análisis de los determinantes que explican que actitudes positivas hacia la adopción concluyan en comportamientos efectivos. La investigación ha subrayado que junto a determinadas características sociodemográficas operan la opinión, actitud y percepción de la norma social de la población respecto al valor que se otorga a la familia, a los hijos y a los modelos familiares tradicionalmente sancionadas. En este ámbito, los datos obtenidos también apuntan diferencias respecto a la población en general. Para los adoptantes, sus hijos ocupan un lugar central en sus familias, por encima de sus parejas; la función principal que asumiría la familia es la de proporcionar amor y afecto a sus hijos; y muestran una mayor tolerancia hacia el modelo de crianza que ofrecen gais y lesbianas. Estos son, a su vez, los elementos que en el orden de los valores se han identificado con la familia postmoderna.

La investigación que sobre las actitudes y las opiniones en relación a la adopción se ha llevado a cabo en países como Estados Unidos, Canadá, Mumbai o Japón ha tomado a la población en general como objeto de estudio. Esta perspectiva ha permitido diferenciar el subgrupo que contemplaría la adopción en su proyecto familiar de entre la población con actitudes positivas hacia la adopción. La encuesta "Las familias adoptivas y sus estilos de vida" se dirigió a madres y padres adoptivos aproximándonos al conjunto de la sociedad a partir de la comparación con los datos ofrecidos por encuestas nacionales. Esta limitación analítica, unido al carácter exploratorio que asume la propia investigación, sugiere la oportunidad de abordar futuras líneas de indagación capaces de describir las opiniones y actitudes de la población española en general hacia la adopción. Dado que en 2013 se presentó el Anteproyecto de Ley de protección a la infancia, con el que se busca potenciar el acogimiento familiar y la adopción abierta, la futura investigación deberá indagar no solo sobre la opinión y actitud de la población frente a la adopción (nacional, internacional, interétnica) sino también ante al acogimiento y la adopción abierta. El conocimiento que derivaría de este tipo de estudios resultaría pertinente tanto para el avance científico como para el desarrollo de políticas públicas fuertemente influenciadas por la opinión pública. En concreto, ofrecería información a la Administración, entidades y profesionales ligados con los procesos de adopción y acogimiento en sus campañas formativas y de reclutamiento. 


\section{BIBLIOGRAFÍA}

ALBERDI, I. y ESCARIO, P. (2003): Flexibilidad, elección y estilos de vida familiar, Madrid, Ministerio de Trabajo y Asuntos Sociales.

ANDERSON, S., PIANTANIDA, M. y ANDERSON C. (1993): "Normal processes in adoptive families", en Normal family processes, Nueva York, Guilford, pp. 254-281.

BAATARD, G. (2012): "A technical Guide to effective and accessible Web surveys", Electronic Journal of Business Research Methods, 10(2), pp.101-109.

BECK, U. y BECK-GERNSHEIM, E. (2001): El normal caos del amor. Las nuevas formas de la relación amorosa, Barcelona, Paidós.

(2003): La individualización. El individualismo institucionalizado y sus consecuencias sociales y políticas, Barcelona, Paidós.

BOSNJAK, M., y TUTEN, T.L. (2003): "Prepaid and promised incentives in web surveys: An experiment“, Social Science Computer Review, 21(2), pp.208-217.

CIS (1995): Encuesta de fecundidad y familia (FFS/ONU). Mujeres, Madrid, CIS, Estudio n ${ }^{\circ} 2182$.

(2004a): Barómetro. Junio 2004, Madrid, CIS, Estudio n².568.

(2004b): Opiniones y actitudes sobre la familia, Madrid, CIS, Estudio n².578.

(2010): Barómetro de septiembre, Madrid, CIS, Estudio n².844.

CHO, H. y LAROSE, R. (1999): "Privacy issues in internet surveys", Social Science Computer Review, 17(4), pp.421-434.

COUPER, M.P. (2000): "Web surveys: a review of issues and approaches", Public Opinion Quarterly, 64(4), pp.464-494.

(2001): Web surveys: the questionnaire design challenge. University of Michigan, Survey Research Center, disponible en http://isi.cbs.nl/iamamember/CD2/pdf/263. PDF) [consulta:12-6-2013].

(2008): Designing effective web surveys, Nueva York, Cambridge University Press. , TRAUGOTT, M.W. y LAMIAS, M.J. (2001): "Web survey design and administration", Public Opinion Quarterly, 65(2), pp. 230-253.

y MILLER, P.V. (2008): "Web survey methods: introduction", Public Opinion Quarterly, 72(5), pp.831-835.

CRAWFORD, S.D., COUPER, M.P. y LAMIAS, M.J. (2001): “Web surveys perceptions of burden”, Social Science Computer Review, 19(2), pp.146-162.

DAVE THOMAS FOUNDATION FOR ADOPTION (2002): National adoption attitudes survey, disponible en http://dciw4f5317k9i.cloudfront.net/wp-content/ uploads/2011/03/2002-Adoption-Exec-Summ.pdf [consulta:25-7-2014].

(2013): National foster care adoption attitudes survey, disponible en http:// dciw4f5317k9i.cloudfront.net/wp-content/uploads/2013/07/DTFA-HarrisPollREPORT-USA-FINALl.pdf [consulta:25-7-2014].

DE LEEUW, E. (2008): "Choosing the method of data collection", en International handbook of survey methodology, Nueva York, Lawrence Erlbaum Associates and European Association of Methodology Series, pp.113-135.

DELGADO, M. (1993): "Cambios recientes en el proceso de formación de la familia", REIS, $n^{\circ}$ 64, pp.125-153.

(coord.) (2007): Encuesta de fecundidad, familia y valores 2006, Madrid, CIS.

DÍAZ DE RADA, V. (2012): "Ventajas e inconvenientes de la encuesta por Internet", Papers, 97(1), pp.193-223.

DILLMAN, D.A. (2000): Mail and web-based surveys: the tailored design method, Nueva York, John Wiley \& Sons. 
CALDWELL, S. y GANSEMER, M. (2000): Visual design effects on item nonresponse to a question about work satisfaction that precedes the Q-12 agreedisagree items, Gallup Research Report, disponible en http://sesrc.wsu.edu/dillman/ papers/2000/visualdesigneffects.pdf [consulta:8-5-2013]

SMYTH, J.D. y CHRISTIAN, L.M. (2009): Internet, mail, and mixed-mode surveys: the tailored design method, Nueva York, John Wiley ( $3^{\mathrm{a} e d .}$.).

FISHBEIN, M. y AJZEN, I. (1975): Belief, attitude, intention, and behavior: an introduction to theory and research, Reading, MA, Addison-Wesley.

GANASSALI, S. (2008): "The influence of the design of web survey questionnaires on the quality of responses", Survey Research Methods, 2(1), pp.21-32.

GR ÄF, L. (2000): "Assessing internet questionnaires: the online pretest lab", en Online Social Sciences, Seattle, WA: Hogrefe \& Huber, pp.73-93.

GROVES, R.M., CIALDINI, R.B. y COUPER, M.P. (1992): “Understanding the decision to participate in a survey", Public Opinion Quarterly, 56(4), pp. 475-495.

GUNN, H. (2002): "Web-based surveys: changing the survey process", First Monday Journal on the Internet, 7(12), disponible en http://www.firstmonday.org/ojs/index. $\mathrm{php} / \mathrm{fm} /$ article/viewArticle/1014/935 [consulta:15-7-2013]

HOKSBERGEN, R.A.C. (1998): "Changes in motivation for adoption, value orientations and behavior in three generations of adoptive parents", Adoption Quarterly, 2(2), pp.37-55.

HOWARD, J. (2012): Untangling the web: the internet's transformative impact on adoption, Evan B. Donalson Adoption Institute, disponible en http:// adoptioninstitute.org/publications/untangling-the-web-the-internets-transformativeimpact-on-adoption/ [consulta:15-7-2014].

ISHIZAWA, H. y KUBO, K. (2013): "Factors affecting adoption decisions: child and parental characteristics", Journal of Family Issues, 35(5), pp.627-653.

JUSTE, MaaG., RAMÍREZ, A. y BARBADILLO, P. (1991): Actitudes y opiniones de los españoles ante la infancia, Madrid, CIS, Estudios y Encuestas, $\mathrm{n}^{\circ} 26$.

KACZMIREK, L. (2005): "Web surveys. A brief guide on usability and implementation issues", en Usability Professionals 2005, German Chapter of the Usability Professionals Association e.V., pp.102-105.

LOZAR, K., BATAGELJ, Z. y VEHOVAR, V. (2002): "Design of web survey questionnaires: three basic experiments", Journal of Computer Mediated Communication, 7(3), disponible en http://jcmc.indiana.edu/vol7/issue3/vehovar. html [consulta:23-9-2013].

LLAURADÓ, O. (2006): "El trabajo de campo online: qué hemos aprendido en los últimos diez años", Investigación y Marketing, n91, pp.25-40.

MALM, K. y WELTI, K. (2010): "Exploring motivations to adopt", Adoption Quarterly, 13(3-4), pp.185-208.

MARRE, D. (2004): "La adopción internacional y las asociaciones de familias adoptantes: un ejemplo de sociedad civil virtual global", Scripta Nova, vol. VIII, no170(4), disponible en http://www.ub.edu/geocrit/sn/sn-170-4.htm [consulta:23-9-2013].

MEIL, G. (1999): La postmodernización de la familia española, Madrid, Acento. (2006): Padres e hijos en la España actual, Barcelona, Obra social "la Caixa". (2011): Individualismo y solidaridad familiar, Barcelona, Obra social "la Caixa".

MIALL, Ch.E. y MARCH, K. (2005): "Social support for changes in adoption practice: gay adoption, open adoption, birth reunions, and the release of confidential identifying information", Families in Society, 86(1), pp.83-92. 
MOHANTY, J. (2013): “Attitudes toward adoption in Singapore”, Journal of Family Issues, 35(5), pp.705-728.

NACIONES UNIDAS (2009): Child adoption: trends and policies, Nueva York, Naciones Unidades.

PARK, N.K. y WONCH, P. (2013): "Is adoption an option? The role of importance of motherhood and fertility help-seeking in considering adoption", Journal of Family Issues, 35(5), pp.601-626.

RAJU, S. (1999): "Study on social attitudes towards child adoption in Munbai", en White paper on adoptions, Catalysts for Social Action (CSA)-Pune, pp.7-10, disponible en http://www.csa.org.in/sites/default/files/WHITE_PAPER_ON_ ADOPTIONS.pdf [consulta:25-7-2014].

RALEIGH, E. (2012): "Are same-sex and single adoptive parents more likely to adopt transracially? A national analysis of race, family structure, and the adoption marketplace", Sociological Perspectives, 55(3), pp. 449-471.

RODRÍGUEZ, J. (2005): Métodos de muestreo. Casos prácticos, Madrid, CIS (2aed.).

RODRÍGUEZ, Ma J. (2012): “Las 'transiciones demográficas' en la segunda modernidad", en Problemas actuales de salud reproductiva, familia, género y sexualidad, Buenos Aires, PNUD/UNFPA y Biblos, pp.83-106.

RUIZ, D. (2004): "Nuevas formas familiares", Portularia, no 4, pp.219-230.

SÁNCHEZ J.J. y SEGOVIA, J.M. (2008): "La participación y la influencia del recordatorio en las encuetas panel on line a estudiantes universitarios", Empiria, nº, pp.135-161.

SELMAN, P. (2006): "Trends in intercountry adoption: analysis of data from 20 receiving countries, 1998-2004", Journal of Population Research, 23(2), pp. 183204.

SHROPSHIRE, K.O., HAWDON, J.E. y WITTE, J.C. (2009): "Web survey design: balancing measurement, response, and topical interest", Sociological Methods and Research, 37(3), pp.343-370.

TOURANGEAU, R., COUPER, M.P. y CONRAD, F. (2004): "Spacing, position, and order. Interpretive heuristics for visual features of survey questions", Public Opinion Quarterly, 68(3), pp.368-393.

TYEBJEE, T. (2003): "Attitude, interest, and motivation for adoption and foster care", Child Welfare, 82(6), pp.685-706.

VAN LANINGHAM, J.L., SCHEUBLE, L.K. y JOHNSON, D.R. (2012): “Social factors predicting women's consideration of adoption", Michigan Family Review, 16(1), pp.1-21.

VICENTE, P. y REIS, E. (2010): "Using questionnaire design to fight nonresponse bias in web surveys", Social Science Computer Review, 28(2), pp.251-267.

YAN, T., CONRAD, F.G., TOUTANGEAU, R. y COUPER, M.O. (2011): "Should I stay or should I go: the effects of progress feedback, promised task duration and length of questionnaire on completing web surveys", International Journal of Public Opinion Research, 23(2), pp.131-147.

YUN, G.W. y TRUMBO, C.W. (2000): "Comparative response to a survey executed by post, e-mail, \& web form", Journal of Computer Mediated Communication, 6(1), disponible en http://onlinelibrary.wiley.com/doi/10.1111/j.1083-6101.2000. tb00112.x/full [consulta:18-10-2013].

WAHL, S.T., MCBRIDE, M.C. y SCHRODT, P. (2005): "Becoming 'point and click' parents: a case study of communication and online adoption", Journal of Family Communication, 5(4), pp.279-294. 